\title{
Effect of the Synthesis Conditions on The Desulfurization Power of Mesoporous Alumina
}

\author{
Dina M. Abd El-Aty, T. Zaki, S. M. Tawfik, O. I. Sif El- \\ Dine, S. I. Hassan and S. H. Ahmed* \\ Petroleum Refining Division, Egyptian Petroleum Research \\ Institute, Nasr City, P.B. 11727 and *Chemistry Department, \\ Faculty of Science, Ain Shams University, Cairo, Egypt.
}

\begin{abstract}
W EsOpOROUS alumina with narrow pore size distribution was synthesized using precipitation method. The effect of various synthesis parameters, including reactants molar ratio, type of co-surfactant, aging time and power source (conventional electric heating (CE) and microwave irradiation (MW)) on the structural properties of the resultant alumina samples were examined. The texture and crystalline phase of the prepared samples were characterized using $\mathrm{N}_{2}$ adsorption-desorption, pyridine adsorption, XRD, TG-DTA, and TEM. The adsorption activity of the prepared samples was examined towards thiophene and dibenzothiophene species.The prepared alumina samples exhibited narrow pore size distribution with high surface area about $262-334 \mathrm{~m}^{2} / \mathrm{g}$, and large mesoporous frameworks with ordered worm-like pores. The preliminary results on the adsorption of sulphur compounds showed that the prepared alumina samples using microwave irradiation as power source and i-propanol as co-surfactant showed distinguished adsorption activities. The structural properties obtained are very interesting for potential applications in desulfurization process in petroleum refining.
\end{abstract}

Keywords: Mesoporous, Alumina, Desulfurization, Adsorption, Dibenzothiophene and Microwave.

Porous materials with a controlled nanostructure are of fundamental importance in science and engineering, and have continued to attract great interest in a variety of applications ${ }^{(1)}$. Different phases of alumina have several properties like high surface area, high thermal, chemical, and mechanical stability, which make it have wide applications in adsorption, separation, and catalysis ${ }^{(2)}$. The disadvantages of uncontrolled porosity such as deactivation by coke formation and plugging of the micropores which hinders the diffusion of reactants and products makes alumina with a narrow pore size distribution, uniform pore structure and high pore volume be highly desirable for practical industrial applications. Synthesis of mesoporous $\mathrm{Al}_{2} \mathrm{O}_{3}$ has been reported using surfactants as templates to direct the formation of mesostructures via interactions between the organic templates and the inorganic precursors, through hydrogen bonding or 
electronic interactions. However, the particle shapes, textural properties and framework crystal phases of mesoporous alumina are related to the preparation conditions ${ }^{(2-3)}$. Depending on the surfactant concentration, the aggregation pattern undergoes changes going from spherical micelles to ellipsoidal, rodlike, or even lamellae, structures. Amphiphilic molecules (as alcohol) often play the role of co-surfactant ${ }^{(4)}$. Within the various methods that are available for the preparation of nanosized oxide powders, precipitation is the most commonly used method not only because it can produce high quality nanopowders but also due to it is cheap. With regarding the source of energy during the precipitation process, microwave heating leads to a more uniform microstructure in comparing with conventional heating. Because of the rapid heating of the microwave process, lower processing temperatures are needed which in turn provides a suitable condition for the formation of nano-sized powders ${ }^{(5)}$. Presence of high levels of sulfur compounds in diesel fuels is still a major source of SOx emission which contributes to air pollution. The primary goal of the recently proposed regulations is to reduce the sulfur content of transportation fuels particularly gasoline and diesel fuels. The sulfur level in these fuels needs to be minimized to reduce the health and environmental hazards. In many countries, the common sulfur specification in diesel is $500 \mathrm{ppm}$; however, some countries have stricter specifications. The new regulations have brought down the sulfur level of diesel from $500 \mathrm{ppm}$ to $50 \mathrm{ppm}$ in many countries while European commission has adopted $10 \mathrm{ppm}$ sulfur specification for the transport diesel ${ }^{(6)}$. In this study, we report the synthesis of mesoporous $\gamma$-alumina by using different power sources and study their effect on the acidity and desulfurization activity of the prepared materials.

\section{Experimental}

\section{Materials}

Aluminium nitrate as aluminium source (Fluka, 98\%), cetyltrimethyl amoniumbromide as structure directing agent (Merck, 99\%), ammonia solution as precipitating agent (Adwic, 30\%), propanol (Riedel-De Hangen AG,99.5\%), iso-propanol (Poch, 99.7\%) and decanol (BDH, 99.7\%) as co-surfactants

\section{Preparation of mesoporous alumina}

Adding the pre-dissolved surfactant to the aluminium nitrate solution under stirring at $1000 \mathrm{rpm}$ and $25^{\circ} \mathrm{C}$, then adding the ammonia solution till stabilize $\mathrm{pH}$ of the solution. The influence of different preparation factors on the nature of resulting materials were studied in this work such as aging time, aluminium/surfactant molar ratio, aluminium/surfactant/co-surfactant molar ratio, type of co-surfactant, time and power of microwave irradiation. The conditions of preparation processes are listed in Table 1 . The precipitates were recovered with centrifugation, washed with distilled water and ethanol 3:1 (Vol./Vol.) at $70{ }^{\circ} \mathrm{C}$ and dried at $80{ }^{\circ} \mathrm{C}$ for $24 \mathrm{hr}$. Calcinations was carried out by heating the assynthesized material to $600{ }^{\circ} \mathrm{C}$ under air flow at a heating rate $1{ }^{\circ} \mathrm{C} / \mathrm{min}$, followed by isothermal heating at $600^{\circ} \mathrm{C}$ for $4 \mathrm{hr}$. For the samples prepared under Egypt. J. Chem. 59, No. 3 (2016) 
microwave irradiation, the calcination process was carried out at $400{ }^{\circ} \mathrm{C}$ for $4 \mathrm{hr}$.

TABLE 1. Conditions of preparation of different alumina samples.

\begin{tabular}{|c|c|c|c|c|c|}
\hline Sample code & \begin{tabular}{|c|} 
metal/ \\
surfactant/ co- \\
surfactant
\end{tabular} & Aging time & co-surfactant & $\begin{array}{c}\text { Heating } \\
\text { type }\end{array}$ & $\begin{array}{l}\text { MW } \\
\text { power }\end{array}$ \\
\hline & molar ratio & $\begin{array}{c}\text { EC (h) - } \\
\text { MW (min) }\end{array}$ & & & $(\mathbf{W})$ \\
\hline $\mathbf{C}_{1 / 1 / 0,2}$ & $1 / 1 / 0$ & 2 & --- & $\mathrm{EC}$ & --- \\
\hline $\mathbf{C}_{1 / 1 / 0,4}$ & $1 / 1 / 0$ & 4 & --- & $\mathrm{EC}$ & --- \\
\hline $\mathbf{C}_{1 / 1 / 0,6}$ & $1 / 1 / 0$ & 6 & --- & $\mathrm{EC}$ & --- \\
\hline $\mathbf{C}_{1 / 1 / 0,10}$ & $1 / 1 / 0$ & 10 & --- & $\mathrm{EC}$ & --- \\
\hline $\mathbf{C}_{0.5 / 1 / 0,4}$ & $0.5 / 1 / 0$ & 4 & --- & $\mathrm{EC}$ & --- \\
\hline $\mathbf{C}_{2 / 1 / 0,4}$ & $2 / 1 / 0$ & 4 & --- & $\mathrm{EC}$ & --- \\
\hline $\mathbf{C}_{3 / 1 / 0,4}$ & $3 / 1 / 0$ & 4 & --- & $\mathrm{EC}$ & --- \\
\hline $\mathbf{C P}_{2 / 1 / 0.5,4}$ & $2 / 1 / 0.5$ & 4 & n-propanol & $\mathrm{EC}$ & --- \\
\hline $\mathbf{C P}_{2 / 1 / 1,4}$ & $2 / 1 / 1$ & 4 & n-propanol & $\mathrm{EC}$ & --- \\
\hline $\mathbf{C P}_{2 / 1 / 2,4}$ & $2 / 1 / 2$ & 4 & n-propanol & $\mathrm{EC}$ & --- \\
\hline $\mathbf{C i P}_{2 / 1 / 1,4}$ & $2 / 1 / 1$ & 4 & Iso-propanol & $\mathrm{EC}$ & --- \\
\hline $\mathbf{C D}_{2 / 1 / 1,4}$ & $2 / 1 / 1$ & 4 & n-decanol & $\mathrm{EC}$ & --- \\
\hline $\mathbf{M}_{2 / 1 / 0, \mathbf{8 , 3}}$ & $2 / 1 / 0$ & 8 & --- & MW & 300 \\
\hline $\mathbf{M P}_{2 / 1 / 1,8,3}$ & $2 / 1 / 1$ & 8 & Propanol & MW & 300 \\
\hline $\mathbf{M i P}_{2 / 1 / 1, \mathbf{8}, 3}$ & $2 / 1 / 1$ & 8 & Iso-propanol & MW & 300 \\
\hline $\mathbf{M D}_{2 / 1 / 1, \mathbf{8 , 3}}$ & $2 / 1 / 1$ & 8 & decanol & MW & 300 \\
\hline $\mathbf{M i P}_{2 / 1 / 1,8,1}$ & $2 / 1 / 1$ & 8 & Iso-propanol & MW & 100 \\
\hline $\mathbf{M i P}_{2 / 1 / 1,8,5}$ & $2 / 1 / 1$ & 8 & Iso-propanol & MW & 500 \\
\hline $\mathbf{M i P} \mathbf{P}_{2 / 1 / 1,8,8}$ & $2 / 1 / 1$ & 8 & Iso-propanol & MW & 800 \\
\hline $\mathbf{M i P}_{2 / 1 / 1,4,3}$ & $2 / 1 / 1$ & 4 & Iso-propanol & MW & 300 \\
\hline $\mathbf{M i P}_{2 / 1 / 1,16,3}$ & $2 / 1 / 1$ & 16 & Iso-propanol & MW & 300 \\
\hline $\mathbf{M i P}_{2 / 1 / 1,24,3}$ & $2 / 1 / 1$ & 24 & Iso-propanol & MW & 300 \\
\hline
\end{tabular}

Sample codes: Heating type, Co-surfactant type, Metal/Surfactant/Co-Surfactant ratio, Aging time, MW Power.

\section{Chracterization}

Differential thermal analysis (DTA) coupled with thermogravimetric analysis (TGA) of the as-synthesised dried powder was recorded on SDT Q600 Simultaneous DSC/TGA Analyzer manufactured by TA Instruments, Inc. (USA). The run was carried out in air at a heating rate of $10^{\circ} \mathrm{C} / \mathrm{min}$. The crystalline phases of the samples were characterized using X-ray diffraction (XRD-6000, Shimadzu, Japan) technique with CuKa radiation of wavelength $\mathrm{k}=0.15418 \mathrm{~nm}$. The microscopic features of the calcined alumina samples were characterized with a transmission electron microscope (TEM) (JEM 2010 from JEOL) operated at $200 \mathrm{kV}$. Nitrogen adsorption/desorption isotherms were measured on a NOVA 3200 analyzer. The samples were degassed at $300{ }^{\circ} \mathrm{C}$ for overnight prior to analysis. The specific surface areas of the samples were calculated using the Brunauer-Emmett-Teller (BET) method in the relative pressure of 0.05-0.35. The pore size distribution (PSD) curves were derived from the desorption branches of the isotherms using the Barrett-Joyner-Halenda (BJH) method. The pore sizes were estimated from the peak positions of the BJH PSD curves. The total pore volumes were estimated from the adsorption quantity at the relative 
pressure of $P / P_{0}=0.993$.

\section{Adsorption activities}

Gas phase pyridine (Py, Sigma-Aldrich, 99.8\%) and thiophene (Thio, SigmaAldrich, 99\%) adsorptions were conducted for all samples. Platinum crucibles loaded with the samples $(50 \mathrm{mg}$ ) were inserted into a glass reactor adapted to a tubular furnace. The materials were dehydrated in dried and purified nitrogen $\left(100 \mathrm{ml} / \mathrm{min}\right.$ ) at $150{ }^{\circ} \mathrm{C}$ for $2 \mathrm{hr}$, cooled to $70^{\circ} \mathrm{C}$ and then gaseous pyridine or thiophene diluted in nitrogen was allowed to pass through the samples for $1.5 \mathrm{hr}$. The temperature was held at $70^{\circ} \mathrm{C}$ under nitrogen for $45 \mathrm{~min}$ to remove physically adsorbed pyridine. After that, the samples were analyzed by TG/DTG ${ }^{(7)}$. The alumina samples that have high activity towards adsorption of thiophene were subjected to adsorption tests towards more bulky sulfur compound; i.e. dibenzothiophene (DBT, Aldrich, 98\%). $100 \mathrm{mg}$ dried alumina sample was mixed with $20 \mathrm{ml}$ of $500 \mathrm{ppm}$ dibenzothiophene/n-dodecane (Merck, $99 \%$ ) solution in adsorption glass bottle. This initial sulfur concentration was selected as a simulation for the concentration of sulfur compounds in the commercial fuels before carrying out the deep desulfurization process ${ }^{(8)}$ and on third of the tri-aromatics in real diesel ${ }^{(9)}$. The well closed bottles were shaking for one hour at $40^{\circ} \mathrm{C}$. The amount adsorbed was determined by analyzing the DBT concentration before and after each experiment using high performance liquid chromatograph (HPLC), manufactured by WATERS, the chromatographic column was of type C18 Microbondaback (300mm x $3.9 \mathrm{~mm})$, which packed with dimethyloctadecylsiltl bonded amorphous silica material (particle size $10 \mu \mathrm{m}$ ). The UV detector (multi-wavelength UV of the type WATERS 490) was adjusted at the wavelength of maximum adsorption, $\lambda_{\max }=254 \mathrm{~nm}$. The influence of adsorbate to adsorbent ratio (volume to mass ratio) on the activity of the highest efficient samples was also studied.

\section{Results and Discussion}

\section{Characterization of mesoporous alumina}

Figure 1 shows DTA plot of as-synthesized sample $\mathbf{C}_{1 / 1 / 0,2}$ after drying at $120^{\circ} \mathrm{C}$. DTA curve of the as-synthesized sample show four endothermic events at ca. $70,285,395$ and $490^{\circ} \mathrm{C}$. The first endothermic event is attributed to the physically adsorbed water. The other endothermic peaks are assigned to dehydroxylation of the sample ${ }^{(1)}$. The DTA curve indicates the absence of any exothermic peak in the range of $400-600^{\circ} \mathrm{C}$, which confirms the success of removing surfactant species using the solvent extraction process. The TG curve shows stepwise weight losses up till $1000^{\circ} \mathrm{C}$. The weight loss $4.8 \%$ at $164^{\circ} \mathrm{C}$ is related to the adsorbed water. The obvious weight loss $21.9 \%$ at $337^{\circ} \mathrm{C}$ may be resulted from the transformation of the aluminium precipitates into $\gamma-\mathrm{Al}_{2} \mathrm{O}_{3}$. The total weight loss in the range $164-1000^{\circ} \mathrm{C}$ is $31.62 \%$. The precipitation of aluminium nitrate using ammonium hydroxide results aluminium hydroxide $\mathrm{Al}(\mathrm{OH})_{3}$ or aluminium monohydrate $\mathrm{AlOOH}$. The theoretical calculation of

weight loss during the thermal treatment showed that the complete Egypt. J. Chem. 59, No. 3 (2016) 
transformation of hydroxide into $\alpha-\mathrm{Al}_{2} \mathrm{O}_{3}$ at temperatures higher than $1000^{\circ} \mathrm{C}$ should be $34.6 \%$. While the theoretical calculation of boehmite $\mathrm{AlOOH}$ transformation is $15.01 \%$. The TG experimental data $(31.62 \%)$ indicates that the precipitate was aluminium hydroxide as major phase in addition to traces of boehmite.

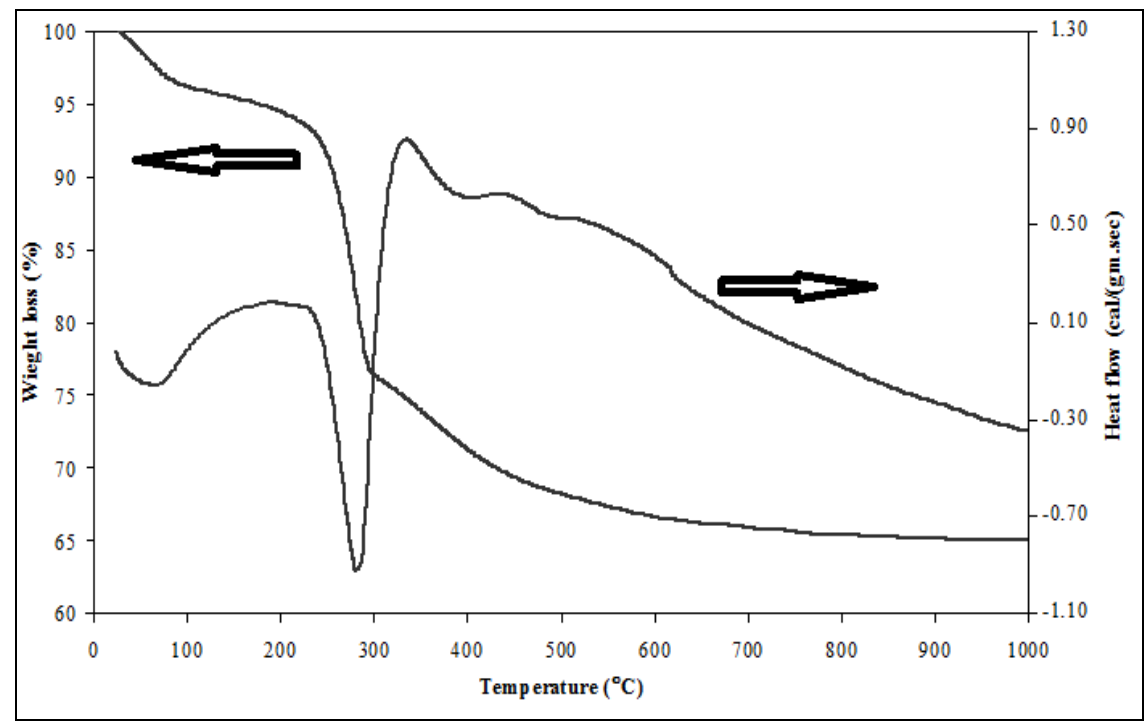

Fig. 1. TG-DTA profiles of sample $C_{1 / 1 / 0,2}$.

Figure 2 showed the XRD patterns of as-synthesized sample $\mathbf{C}_{1 / 1 / 0,2}$ calcined at different temperatures. The dried as-synthesized sample shows the reflections of highly crystalline aluminium hydroxide or Bayerite in agreement with the interpretation of TG analysis (JCPDS file 01-074-1119) Figure 2a. The XRD patterns of the sample after calcination at temperatures 165 and $220{ }^{\circ} \mathrm{C}$ indicate the presence of Bayerite as major phase in spite the decrease in the intensity of the reflection as the calcination temperature increased (Fig. $2 b$ and $2 c$ ). Upon calcination process at $390{ }^{\circ} \mathrm{C}$, the reflections related to $\eta$-alumina become the predominant in the pattern at d-spaces $1.977,1.398$ and $2.384 \AA$ (JCPDS file 01077-0396) (Fig. 2d instead of those of Bayerite). The reflections of $\gamma$-alumina appeared after calcination at temperature $600{ }^{\circ} \mathrm{C}$ at d-spaces $1.975,1.397$ and $2.281 \AA$ (JCPDS file 01-075-0921) (Fig. 2e in addition to the reflections of $\eta$ alumina). All prepared alumina samples using different preparation conditions and conventional electric heating process show similar XRD patterns. On the other hand, the alumina samples that prepared under the microwave irradiation showed $\gamma$-alumina reflections at d-spaces $1.975,1.397$ and $2.281 \AA$ (JCPDS file 01-075-0921) (Fig. 3a after calcination at temperature $400^{\circ} \mathrm{C}$ ). 


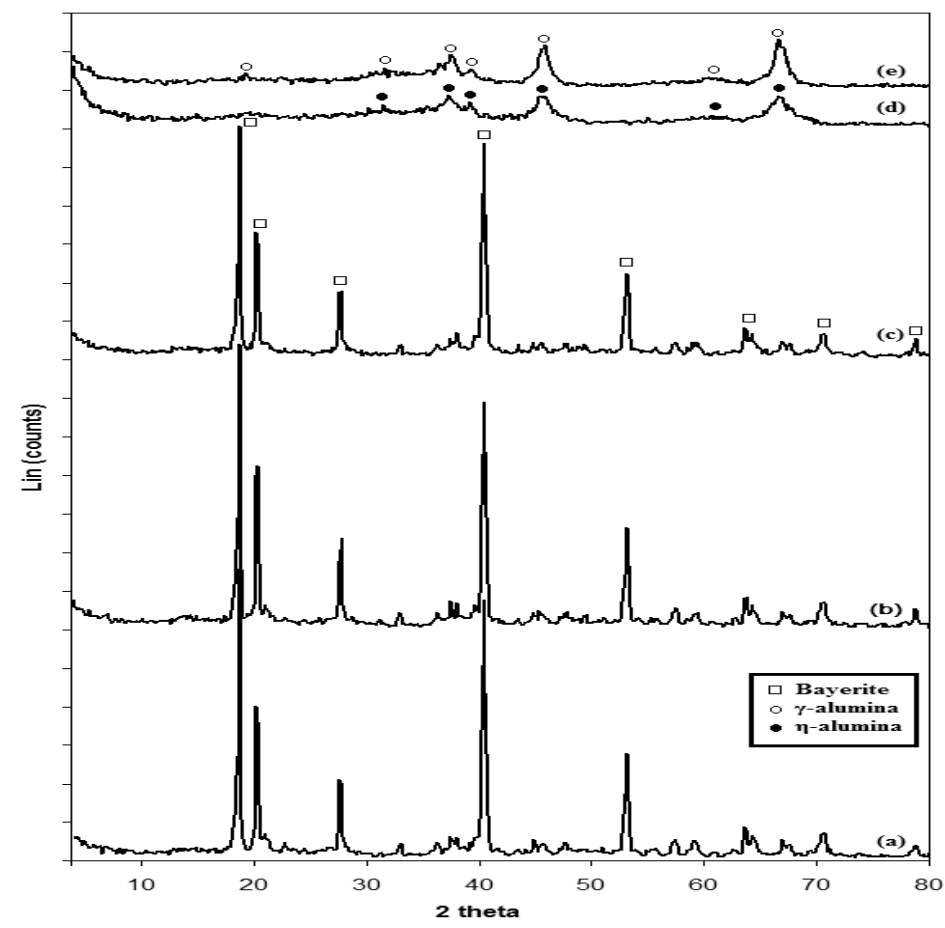

Fig. 2. $X$-ray diffraction patterns of (a) dried sample $C_{1 / 1 / 0,2}$ and calcined at (b) 165 , (c) 220 , (d) 390, and (e) $600{ }^{\circ} \mathrm{C}$.

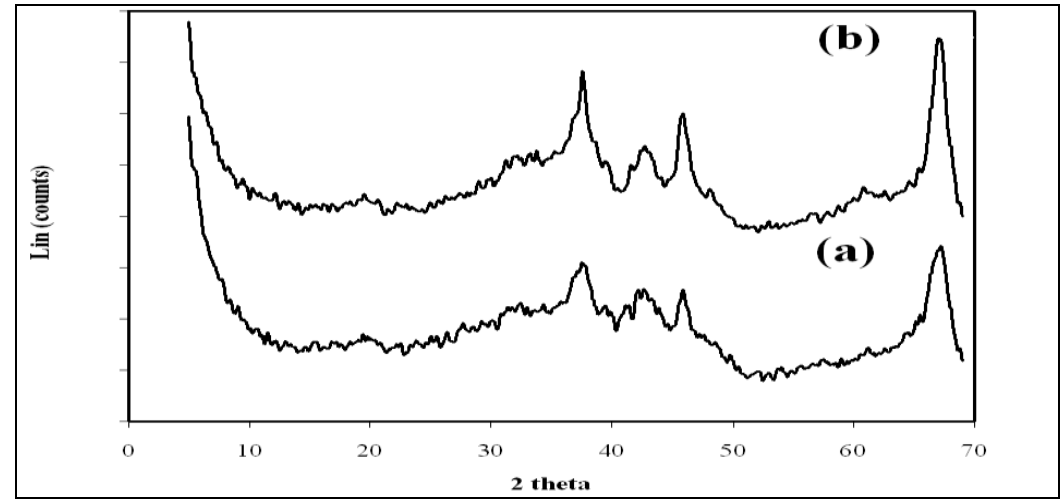

Fig. 3. X-ray diffraction patterns for (a) $\mathrm{MiP}_{2 / 1 / 1,8,3}$, and (b) $\mathrm{C}_{1 / 1 / 1,2}$ samples.

Nitrogen adsorption - desorption isotherms at liquid nitrogen temperature $\left(-196^{\circ} \mathrm{C}\right)$ for the prepared alumina were found to have reversible isotherms exhibiting type IV (Fig. 4-5) according to the IUPAC classification, indicating the existence of mesopore structures. The hysteresis loops of nitrogen sorption isotherms are typical or textural porosity ${ }^{(2)}$. The isotherms of alumina samples that prepared Egypt. J. Chem. 59, No. 3 (2016) 
using conventional electric heating exhibiting close hysteresis loops of type H3 (Fig. 4a), while the isotherms of alumina samples synthesized via the microwave irradiation showed close hysteresis loops of type H2 (Fig. 5a). The hysteresis loop $\left(\mathbf{C}_{2 / 1 / 0,4}\right)$ sample (Fig. $\left.4 a\right)$ at $\mathrm{P} / \mathrm{P0}$ value of $0.4-0.8$ is due to the capillary condensation within the uniform mesopores. On the other hand, hysteresis loops of type $\mathrm{H} 2$ (Fig. 5a) represents a mesostructure with narrow pore size distributions ${ }^{(3)}$. The $\mathrm{H} 3$ hysteresis loop indicates the presence of non-rigid aggregates (assemblage of particles which are loosely coherent) of plate-like particles giving rise to slit-shaped pores ${ }^{(10-12)}$. On the other hand, the H2 hysteresis loops are generally taken as an indication of "bottle-neck" like pore structures. The textural properties of the prepared alumina samples are listed in Table 2. It was found that the alumina samples get the optimum surface area $219 \mathrm{~m}^{2} / \mathrm{g}\left(\mathbf{C}_{1 / 1 / 0,4}\right)$, after aging time four hours, which has the narrow pore radius $\sim 18 \AA$. At the optimum aging time, the surface area increases slightly as the metal salt/surfactant molar ratio increases from $1: 1$ to $2: 1$, but no significant changes were appeared for the values of the total pore volume and average pore radius. Additional increase in the metal salt/surfactant molar ratio up to $3: 1$ showed decreasing in the surface area from $\sim 224 \mathrm{~m}^{2} / \mathrm{g}\left(\mathbf{C}_{2 / 1 / 0,4}\right)$ to $\sim 205 \mathrm{~m}^{2} / \mathrm{g}$ $\left(\mathbf{C}_{3 / 1 / 0,4}\right)$.

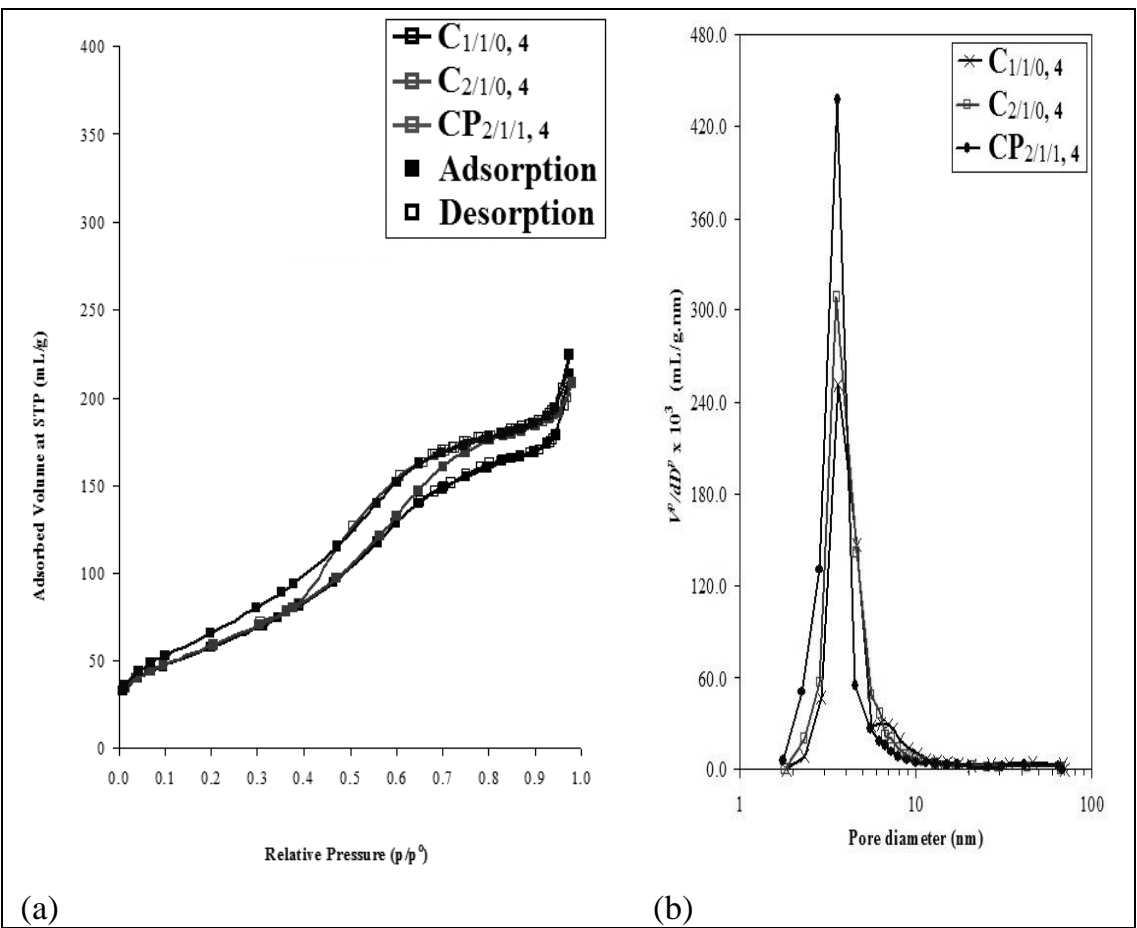

Fig. 4. (a) Nitrogen adsorption-desorption isotherms and (b) BJH desorption pore size distributions of selected samples prepared via conventional electric heating. 
The presence of co-surfactant in the solution does not induce an observable modification in the pore diameter but improve the surface area. The using of npropanol as co-surfactant enhances the surface area of the prepared alumina samples, whereas increased from $\sim 224 \mathrm{~m}^{2} / \mathrm{g}\left(\mathrm{C}_{2 / 1 / 0,4}\right)$ to $\sim 239 \mathrm{~m}^{2} / \mathrm{g}\left(\mathrm{CP}_{2 / 1 / 0.5,4}\right)$. Upon increasing the metal salt/surfactant/co-surfactant molar ratio to $2 / 1 / 1$ $\left(\mathrm{CP}_{2 / 1 / 1,4}\right)$ the surface area of the prepared alumina reached its maximum value $262 \mathrm{~m}^{2} / \mathrm{g}$ (Table 2). Further increasing in the co-surfactant molar ratio leads to surface area depression down to $\sim 209 \mathrm{~m}^{2} / \mathrm{g}\left(\mathbf{C P}_{2 / 1 / 2,4}\right)$. The BJH desorption pore size distributions (Fig. $4 b)$ clarified that the $\left(\mathbf{C}_{1 / 1 / 0,4}\right),\left(\mathbf{C}_{2 / 1 / 0,4}\right)$ and $\left(\mathbf{C P}_{2 / 1 / 1,4}\right)$ samples have mono - type of mesopores. The pore diameter has value of about $3.6 \mathrm{~nm}$, indicating that the samples have uniform pore size and ordered mesostructure. However $\left(\mathbf{C P}_{2 / 1 / 1}, 4\right)$ sample showed the highest amount of mesopores. Commutation the n-propanol with branched alcohol such as iso-propanol or bulky alcohol such as n-decanol does not improve the surface features. The data listed in Table 2 clarified that the shape of the co-surfactant affect only on surface area and pore volume but not on pore diameter.

TABLE 2. Surface textural properties of alumina samples.

\begin{tabular}{|c|c|c|c|c|}
\hline \multirow[t]{2}{*}{ Sample code } & $\begin{array}{c}\text { S }_{\text {BET }} \\
\text { BET } \\
\text { surface } \\
\text { area } \\
\end{array}$ & $\begin{array}{c}V_{p} \\
\text { total pore } \\
\text { volume }\end{array}$ & $\begin{array}{c}\mathrm{V}_{\text {p-micro }} \\
\text { micropores } \\
\text { volume }\end{array}$ & $\begin{array}{c}\mathbf{r}_{H} \\
\text { average pore } \\
\text { radius }\end{array}$ \\
\hline & $\left(\mathrm{m}^{2} / \mathrm{g}\right)$ & $\left(\mathrm{cm}^{3} / \mathrm{g}\right)$ & $\left(\mathrm{cm}^{3} / \mathrm{g}\right)$ & $\AA$ \\
\hline $\mathbf{C}_{1 / 1 / 0,2}$ & 202.51 & 0.377 & --- & 21.67 \\
\hline $\mathbf{C}_{1 / 1 / 0,4}$ & 218.50 & 0.357 & 0.023 & 18.05 \\
\hline $\mathbf{C}_{1 / 1 / 0,6}$ & 210.90 & 0.400 & --- & 18.10 \\
\hline $\mathbf{C}_{1 / 1 / 0,10}$ & 175.60 & 0.266 & --- & 21.64 \\
\hline $\mathbf{C}_{0.5 / 1 / 0,4}$ & 217.50 & 0.435 & --- & 18.16 \\
\hline $\mathbf{C}_{2 / 1 / 0,4}$ & 223.50 & 0.346 & 0.023 & 18.01 \\
\hline $\mathbf{C}_{3 / 1 / 0,4}$ & 204.70 & 0.322 & 0.025 & 18.02 \\
\hline $\mathbf{C P}_{2 / 1 / 0.5,4}$ & 236.30 & 0.520 & 0.003 & 17.93 \\
\hline $\mathbf{C P}_{2 / 1 / 1,4}$ & 262.30 & 0.380 & 0.067 & 18.08 \\
\hline $\mathbf{C P}_{2 / 1 / 2,4}$ & 209.00 & 0.463 & 0.010 & 18.04 \\
\hline $\mathbf{C i P}_{2 / 1 / 1,4}$ & 197.90 & 0.744 & --- & 18.04 \\
\hline $\mathbf{C D}_{2 / 1 / 1,4}$ & 235.80 & 0.433 & 0.012 & 18.14 \\
\hline $\mathbf{M}_{2 / 1 / 0,8,3}$ & 308.20 & 0.500 & --- & 28.40 \\
\hline $\mathbf{M P} \mathbf{P}_{2 / 1 / 1,8,3}$ & 293.50 & 0.486 & --- & 28.27 \\
\hline $\mathbf{M i P}_{2 / 1 / 1,8,3}$ & 334.20 & 0.535 & --- & 23.21 \\
\hline $\mathbf{M D}_{2 / 1 / 1, \mathbf{8}, \mathbf{3}}$ & 268.10 & 0.526 & --- & 28.48 \\
\hline $\mathbf{M i P}_{2 / 1 / 1,8,1}$ & 266.20 & 0.368 & 0.037 & 17.97 \\
\hline $\mathbf{M i P}_{2 / 1 / 1, \mathbf{8}, \mathbf{5}}$ & 291.50 & 0.519 & --- & 28.48 \\
\hline $\mathbf{M i P}_{2 / 1 / 1, \mathbf{8}, \mathbf{8}}$ & 274.50 & 0.429 & 0.023 & 23.26 \\
\hline $\mathbf{M i P}_{2 / 1 / 1,4,3}$ & 276.70 & 0.480 & --- & 34.34 \\
\hline $\mathbf{M i P}_{2 / 1 / 1,16,3}$ & 314.80 & 0.543 & --- & 23.34 \\
\hline $\mathbf{M i P}_{2 / 1 / 1,24,3}$ & 258.70 & 0.423 & --- & 28.43 \\
\hline
\end{tabular}

The changing of energy source during the preparation process from conventional electric heating to microwave irradiation provokes dramatically improvement in the surface features of the prepared alumina samples. $\left(\mathrm{M}_{2 / 1 / 0,8,3}\right)$ sample showed obvious high surface area $\sim 308 \mathrm{~m}^{2} / \mathrm{g}$, and wider pore radius $\sim 28$ $\AA$, with respect to the alumina sample prepared using similar chemical composition of reactants $\left(\mathbf{C}_{2 / 1 / 0,4}\right)$. Such enhancing in the surface properties Egypt. J. Chem. 59, No. 3 (2016) 
accomplished with severe reduce in aging time from four hours to eight minutes. In contrary with the conventional electric heating process, the branching in the alcohol structure improve the surface area to $\sim 315 \mathrm{~m}^{2} / \mathrm{g}\left(\mathbf{M i P}_{\mathbf{2} / 1 / 1, \mathbf{8}, \mathbf{3}}\right)$. The using of normal alcohol as co-surfactant either small molecule, i.e. n-propanol or large molecule such as n-decanol acted negatively on the surface area of the prepared alumina under similar experimental circumstances. $\left(\mathbf{M P} \mathbf{P}_{2 / 1 / 1, \mathbf{8 , 3}}\right)$ and $\left(\mathbf{M D}_{2 / 1 / 1, \mathbf{8 , 3}}\right)$ samples exhibited surface area $\sim 294$ and $\sim 268 \mathrm{~m}^{2} / \mathrm{g}$, respectively, which are lower than the surface area of the alumina sample prepared without using cosurfactant. The surface data that are listed in Table 2 revealed that the microwave power ( $300 \mathrm{watt}$ ) is the optimum energy for the preparation process. Also, $8 \mathrm{~min}$. aging time results the alumina sample of the highest surface area in this study $334 \mathrm{~m}^{2} / \mathrm{g}\left(\mathbf{M i P}_{2 / 1 / 1, \mathbf{8}, 3}\right)$, big total pore volume $0.535 \mathrm{~cm}^{3} / \mathrm{g}$ and moderate average pore radius $\sim 23 \AA$. The relatively low surface area values of the alumina samples prepared using the conventional electric heating process in comparison to the surface area values for the samples prepared using microwave irradiation (Table 2) could be attributed to the influence of the calcination temperature that used during the preparation process. Whereas the first category of samples calcined at $600^{\circ} \mathrm{C}$ while the second category prepared after the calcination process at $400^{\circ} \mathrm{C}$.

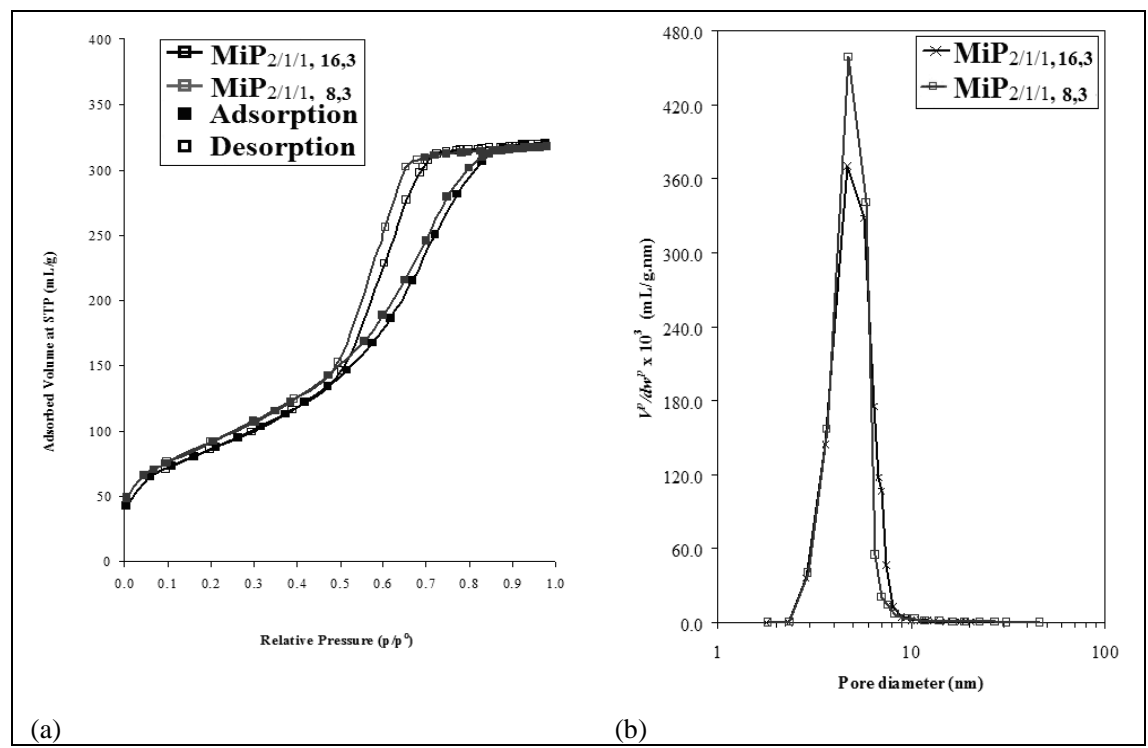

Fig. 5(a) Nitrogen adsorption-desorption isotherms and (b) BJH desorption pore size distributions of selected samples prepared via microwave irradiation.

This assumption based on the influence of the calcination temperature on the creation of mesopores. Whereas large number of small mesopores, initially present in the as-synthesized materials, transform by coalescence at high temperature into a restricted number of very large mesopores (through some pore wall breaking), giving rise large mesoporous volume but also small surface area ${ }^{(13)}$. In general the previous mentioned experimental data could be related to 
the existence of the surfactant micelles in the synthesis medium, which is a crucial point as it affects the ordering of the finally formed templated mesophase. Considering the cationic charge of the template and the aluminium species present at low $\mathrm{pH}$ (below 3), an electrostatic self-assembly pathway with bridging halide species seems to be the more feasible formation mechanism. The synthesis conditions also determine the nature of the different aluminium hydroxides or oxo-hydroxides (amorphous, crystalline) which make up the pore walls and their respective condensation degree ${ }^{(14)}$.

Figure 6 illustrates the proportional relation between the values of surface area and the surface acidity of the prepared alumina samples under different preparation conditions. Whereas $\mathbf{C}_{2 / 1 / 0,4}$ exhibits high pyridine adsorption (1285 $\mu \mathrm{mol}$ pyridine/g) among the samples prepared using different metal precursor to surfactant ratio, also the same sample showed high surface area $\left(223.5 \mathrm{~m}^{2} / \mathrm{g}\right)$ (Fig. 5a). Figure 6b showed slight increase in the pyridine adsorption capacity as the molar ratio of the n-propanol increase $\left(\mathbf{C P}_{2 / 1 / 1}, 4,1302 \mu \mathrm{mol}\right.$ pyridine/g), instead of the obvious enhancement in the surface area from $223.5 \mathrm{~m}^{2} / \mathrm{g}\left(\mathbf{C}_{2 / 1 / 0,4}\right)$ to $262.3 \mathrm{~m}^{2} / \mathrm{g}\left(\mathbf{C P}_{2 / 1 / 1,4}\right)$. The using of iso-propanol and $\mathrm{n}$-decanol as cosurfactant caused decrease in the surface acidity to 1178 and $1273 \mu \mathrm{mol}$ pyridine/g respectively (Fig. 6c). On the other hand, the using of iso-propanol under the irradiation of microwave causes opposite effect on the surface area

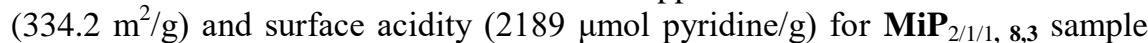
with respect to the alumina samples prepared without using co-surfactant $\left(\mathbf{M}_{2 / 1 / 0}\right.$ $\mathbf{8 , 3}, 308.2 \mathrm{~m}^{2} / \mathrm{g}, 1999 \mu \mathrm{mol}$ pyridine/g) or with using n-propanol as co-surfactant $\left(\mathbf{M P}_{2 / 1 / 1, \mathbf{8 , 3}}, 293.50 \mathrm{~m}^{2} / \mathrm{g}, 1920 \mu \mathrm{mol}\right.$ pyridine/g) (Fig. 6d).

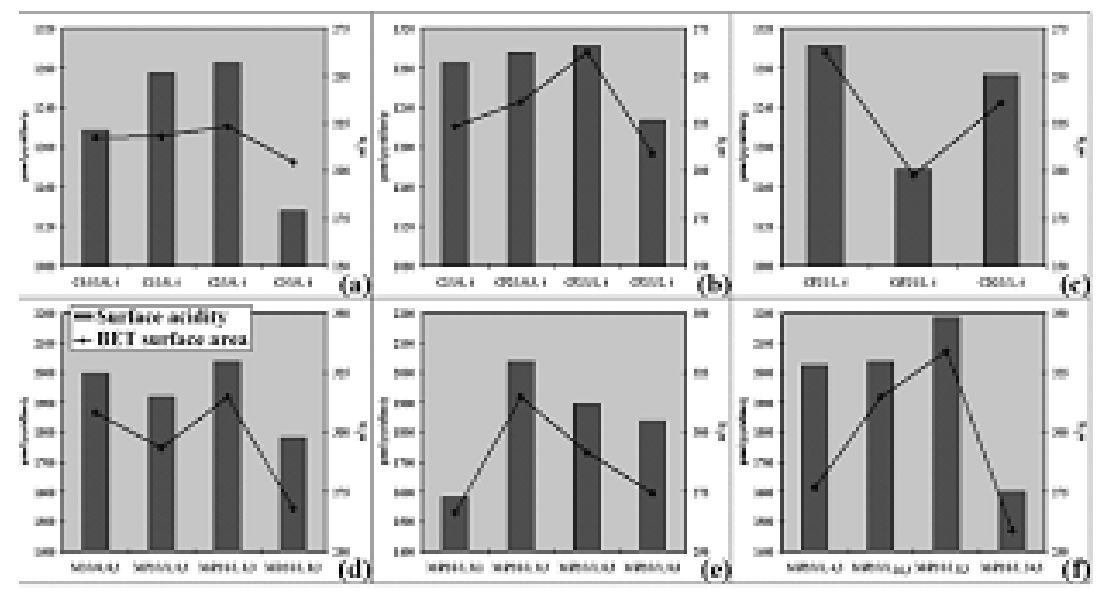

Fig. 6. Relation between the BET surface area $\left(\mathrm{m}^{2} / \mathrm{gm}\right)$ and surface acidity using pyridine adsorption $(\mu \mathrm{mol} / \mathrm{gm})$ for different prepared alumina samples.

Regarding the influence of the microwave irradiation power on the surface features of the prepared alumina samples, the microwave power (300 watt) was found to be the optimum energy that required for the lowest value of aging time Egypt. J. Chem. 59, No. 3 (2016) 
surface area and surface acidity. However, the suffering of alumina sample to the minimal value of power (100 watt) for $8 \mathrm{~min}$. (MiP $\mathbf{M}_{2 / 1 / 1,8,1}$ sample) showed depression in both of surface area $\left(266.2 \mathrm{~m}^{2} / \mathrm{g}\right)$ and surface acidity $(1582 \mu \mathrm{mol}$ pyridine/g) as appeared in Fig. 6e. The prolongation of aging time under the irradiation of microwave to $16 \mathrm{~min}$. showed depression in the values of the surface area $\left(314.8 \mathrm{~m}^{2} / \mathrm{g}\right)$ and surface acidity $(2037 \mu \mathrm{mol}$ pyridine $/ \mathrm{g})$, respectively Figure $6 \mathrm{f}$ and upon more extension in the aging time under the irradiation of microwave to $24 \mathrm{~min}$. caused sharp decrease in both of surface area $\left(258.7 \mathrm{~m}^{2} / \mathrm{g}\right)$ and surface acidity (1599 $\mu \mathrm{mol}$ pyridine/g) successively (Fig. 6f).

The TEM images (Fig. 7) show the rod-like shape of the $\gamma$-alumina samples prepared either by using conventional electric heating source, i.e. $\mathbf{C P}_{2 / 1 / 1,4}$ sample (Fig. 7) or using microwave irradiation source. It is well known that depending on the surfactant concentration, the aggregation pattern undergoes changes going from spherical micelles to ellipsoidal, rod-like, or even lamellae, structures. Amphiphilic molecules (as alcohol) often play the role of co-surfactant. Partitioned between aqueous and micellar phases, they act upon micellar structures where the amphiphilic molecule shields the polar head groups of the surfactant at the micellar interface which diminishes the repulsive interactions between charged heads. With amphiphilic long molecules (long-chain alcohols or hydrosoluble polymers), the viscosity increases progressively with increasing concentration or decreasing temperature. With particular amphiphilic solutes, the viscosity reveals a great sensitivity to a small variation of solute concentration or to a weak temperature change. In this case, strong interactions between the polar head groups of the surfactant and the solute promote the formation of worm-like micelles ${ }^{(4)}$.

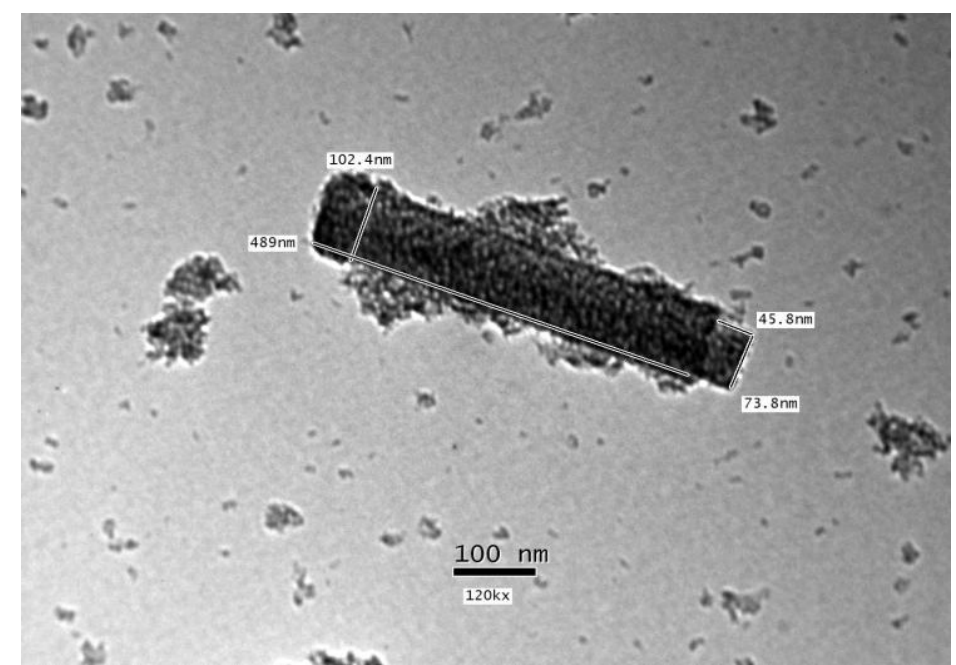

Fig. 7. TEM micrograph $\mathrm{CP}_{2 / 1 / 1,4}$ sample. 


\section{Thiophene adsorption}

Figure 8 illustrates the adsorption capacity of different prepared alumina samples towards the thiophene species in nitrogen gas stream. Also, the molar ratio between the adsorbed pyridine species and the adsorbed thiophene species for each sample under the same experimental conditions can be seen in Fig.8. In general it seems that there is a positive proportional relation between the adsorption capacity of the alumina samples and their surface area. Due to the mesoporous structure of all prepared samples, the influence of thiophene diffusion (critical diameter $5.3 \AA$ ) seems to be not one of the control factors in this study ${ }^{(15)}$. Also, it is obvious that all alumina samples that prepared under the irradiation of microwave (Fig 8d-f) showed higher thiophene adsorption capacity than other samples prepared via the conventional electric heating process (Fig 8ac). Such high capacity could be attributed to that the particles size of alumina samples prepared via microwave are relatively smaller than that prepared via the conventional electric heating process. The decreasing in the particle size meaning that there is increase in the amount of edges and corners among the amount of the used adsorbent, which successively means increase in the amount of active sites. The third attractive point that can be seen in Fig. 8 is that there contrary proportional relation between the adsorption capacity of the alumina samples towards the thiophene species and the molar ratio between the adsorbed pyridine species and the adsorbed thiophene species by each sample. This relation could be explained through the affinity of the adsorbent species towards the acid sites in the surface of the adsorbate. It is well known that the lone pair of electron on sulfur atom or $\pi$-electrons in the aromatic ring of thiophene transferred favorably to Lewis acid sites to form S-bonding or $\pi$-type complex ${ }^{(15)}$.

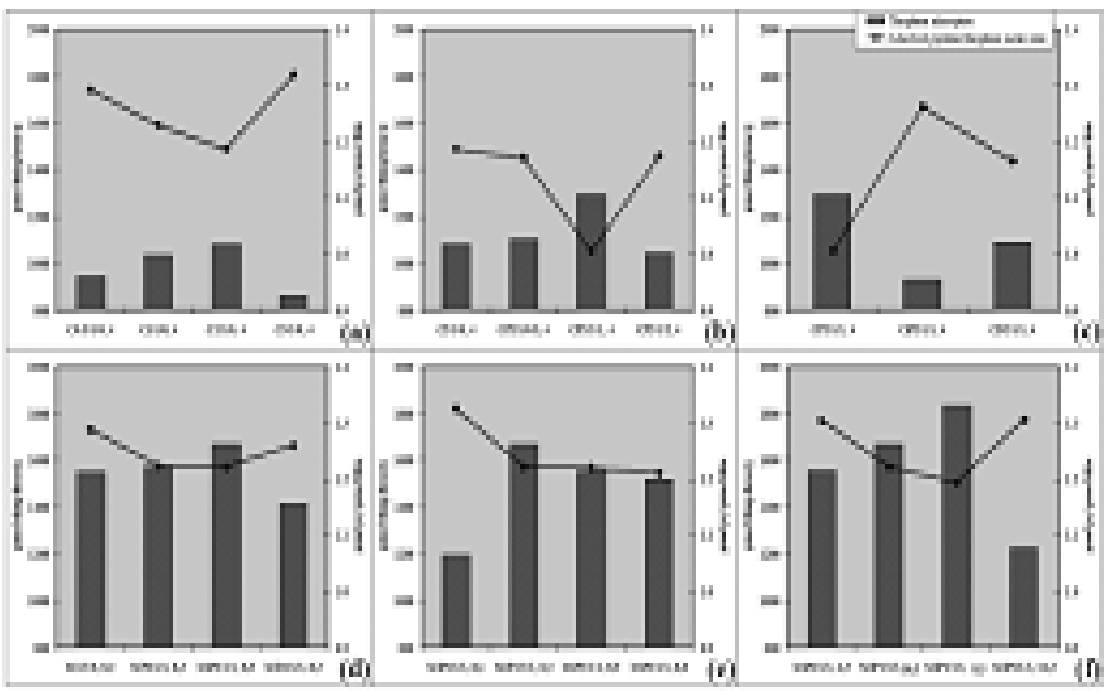

Fig. 8. Thiophene and pyridine adsorption capacity of different prepared alumina samples.

Egypt. J. Chem. 59, No. 3 (2016) 
Whereas the pyridine molecules have more delocalized electrons than thiophene molecules, accordingly it should be expected that the pyridine species favor any type of acid site, i.e. strong, medium or weak acid sites, while thiophene species favor the stronger acid sites. According the previous adsorption experimental data, we can conclude that $\mathbf{C P}_{2 / 1 / 1,4}$ and $\mathbf{M i} \mathbf{P}_{2 / 1 / 1}, \mathbf{8 , 3}$ samples have the highest surface area 262.30 and $334.20 \mathrm{~m}^{2} / \mathrm{g}$, respectively and the largest amount of the strong acid sites.

\section{Dibenzothiophene adsorption}

The transport of adsorbate from the solution phase into the pores of the adsorbent particles may be controlled either by one or more steps, e.g. film or external diffusion, pore diffusion, surface diffusion and adsorption on the pore surface, or a combination of more than one step. For adsorption process, the external mass transfer controls the sorption process for the systems that have poor mixing, dilute concentration of adsorbate, small particle sizes of adsorbent and higher affinity of adsorbate for adsorbent. Whereas, the intra-particle diffusion controls the adsorption process for a system with good mixing, large particle sizes of adsorbent, high concentration of adsorbate and low affinity of adsorbate for adsorbent ${ }^{(16)}$. The DBT molecule, a three-ring aromatics with a sulfur atom, the two unpaired electrons of the sulfur atom in DBT act as an electron donor to acid sites, in which the electron-withdrawing groups acting as an electron acceptor ${ }^{(9)}$. (Fig. 9) showed the adsorption capacity of different prepared alumina samples towards the dibenzothiophene species dissolved in $\mathrm{n}$ heptane $(500 \mathrm{ppm})$. Among the alumina samples that prepared using conventional electric heating, $\mathbf{C P}_{2 / 1 / 1,4}$ sample achieved the highest adsorption activity 78.71 $\mu \mathrm{mole} / \mathrm{g}$. The adsorption activity shows direct proportional relation with the values of the surface area and strength of the acid sites on the surfaces of the tested samples. No noticeable influence for the average pore radius was detected, whereas the average diameter of the pores on the surfaces of all samples is suitable with respect to the critical diameter of DBT species ( $8 \AA$ ) or the area of the DBT $8 \times 12 \AA^{2}{ }^{(9)}$. On the other hand, the alumina samples that prepared using microwave irradiation showed higher adsorption activities than the other samples.

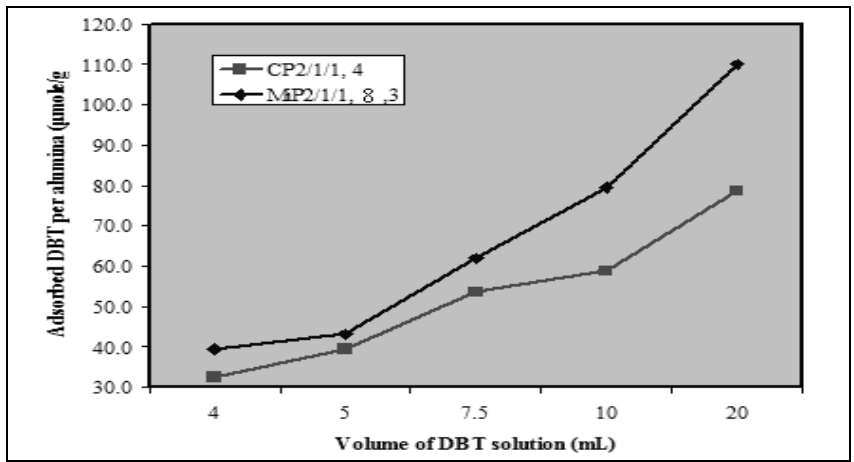

Fig. 9. Relation between the adsorption capacity $(\mu \mathrm{mole} / \mathrm{g})$ and the volume of DBT solution (mL) for $\mathrm{CP}_{2 / 1 / 1,4}$ and $\mathrm{MiP}_{2 / 1 / 1,16,3}$ samples. 
This adsorption behaviour could be attributed to the relatively large average pore radius for these samples (ranged from $\sim 23$-to $\sim 34 \AA$ ) with respect to the alumina samples prepared using conventional electric heating $(\sim 18 \AA)$. The sample $\mathbf{M i P}_{2 / 1 / 1,8,3}$ achieved the highest adsorption capacity $(110.14 \mu \mathrm{mole} / \mathrm{g})$ with respect to all of the prepared samples.

\section{Conclusions}

Mesoporous $\gamma$-aluminas were synthesized by precipitation method with various synthesis parameters, including reactant molar ratio, type of cosurfactant, aging time and power source (conventional electrical heating and microwave irradiation). It was found that the prepared mesoporous aluminas by using microwave irradiation have larger surface areas, pore volumes and narrow pore size distributions than those prepared by conventional electrical heating method.

These are anticipated to be excellent candidates as sulfur adsorbent materials because of the uniform pore structure, large surface areas, narrow pore size distribution and large quantities of surface lewis acid sites.

\section{References}

1. Kim, Y., Kim, C., Kim, P. and Yi, J., Effect of preparation conditions on the phase transformation of mesoporous alumina. Journal of Non-Crystalline Solids , 351, 550$556(2005)$

2. Li, D.Y., Lin, Y.S., Li, Y.C., Shieh, D.L. and Lin, J.L., Synthesis of mesoporous pseudoboehmite and alumina templated with 1-hexadecyl-2,3-dimethyl-imidazolium chloride. Microporous and Mesoporous Materials, 108, 276-282 (2008) .

3. Liu, Q., Wang, A., Wang, X. and Zhang, T., Mesoporous $\gamma$-alumina synthesized by hydro-carboxylic acid as structure-directing agent. Microporous and Mesoporous Materials , 92, 10-21(2006).

4. Busserolles, K., Roux-Desgranges, G. and Roux, A.H., Thermodynamic study in aqueous micellar solutions at $298.15 \mathrm{~K}$ : comparison of the interactions between ionic surfactants (SDS and CTAB) and 1-alcohols or phenol. Thermochimica Acta, 259, 4956 (1995).

5. Ebadzadeh, T. and Asadian, K., Microwave-assisted synthesis of nanosized $\alpha-\mathrm{Al}_{2} \mathrm{O}_{3}$. Powder Technology, 192, 242-244 (2009).

6. Sarda, K.K., Bhandari, A., Pant, K.K. and Jain, S. Deep desulfurization of diesel fuel by selective adsorption over $\mathrm{Ni} / \mathrm{Al}_{2} \mathrm{O}_{3}$ and Ni/ZSM-5 extrudates. Fuel, 93, 86-91 (2012).

7 Ghesti, G.F., de Macedo, J.L., Parente, V.C.I., Dias, J. A. and Dias, Sı 'Ivia C.L., Controlling the textural parameters of mesoporous carbon materials. Microporous and Mesoporous Materials, 100, 27-34 (2007).

Egypt. J. Chem. 59, No. 3 (2016) 
8. Ma, X., Velu, S., Kim, J.H. and Song, C., Deep desulfurization of gasoline by selective adsorption over solid adsorbents and impact of analytical methods on ppmlevel sulfur quantification for fuel cell applications. Applied Catalysis B: Environmental, 56, 137-147(2005).

9. Bu, J., Loh, G., Gwie, C.G., Dewiyanti, S., Tasrif, M. and Borgna, A., Desulfurization of diesel fuels by selective adsorption on activated carbons: Competitive adsorption of polycyclic aromatic sulfur heterocycles and polycyclic aromatic hydrocarbons. Chemical Engineering Journal, 166 , 207-217 (2011).

10. Zainab Ramli and Sheela Chandren, Effect of templates on the synthesis of organized meso porous alumina. The Malaysian Journal of Analytical Sciences, 11, $110-116$ (2007).

11. Lowell, S., Shields, J.E., Thomas, S.M.A., Martin, S.A. and Matthias, Thommes, Kluwer Academic Publishers, London (2004).

12. Gregg, S.J. and Sing, K.S.W., "Adsorption Surface Area and Porosity", $2^{\text {nd }}$ ed., Academic Press, Britain (1982).

13. Valange, S., Guth, J.L., Kolenda, F., Lacombe, S. and Gabelica, Z., Synthesis strategies leading to surfactant-assisted aluminas with controlled mesoporosity in aqueous media. Microporous and Mesoporous Materials, 35-36, 597-607 (2000).

14. Aguado, J., Escola, J.M., Castro, M.C. and Paredes, B., Sol-gel synthesis of mesostructured $\gamma$-alumina templated by cationic surfactants. Microporous and Mesoporous Materials, 83, 181-192 (2005).

15. Subhan, F. and Liu, B.S., Acidic sites and deep desulfurization performance of nickel supported mesoporous AlMCM-41 sorbents. Chemical Engineering Journal, 178, 69- 77 (2011).

16. Srivastav, A. and Srivastava, V.C., Adsorptive desulfurization by activated alumina. Journal of Hazardous Materials, 170, 1133-1140 (2009).

(Received 26/1/2016, accepted 2 / 3/2016) 


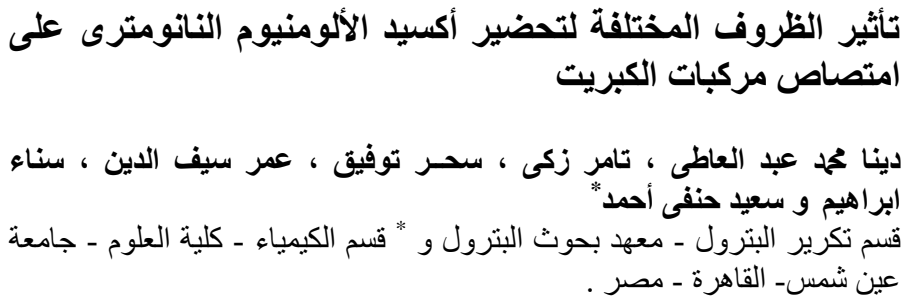

تم تحضير أكسيد الألومنيوم النانومترى ذو حجم الفجوات القريبة من بعضها

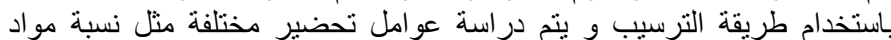

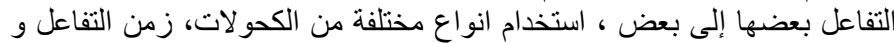

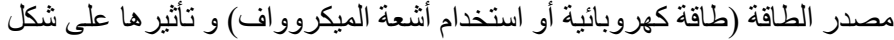

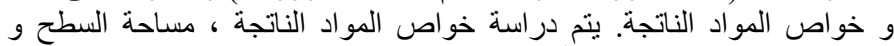

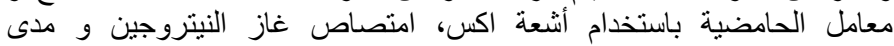

امتصاص مادة قاعدية مثل البيريدين.

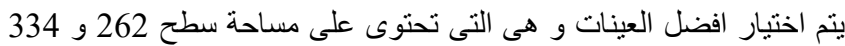

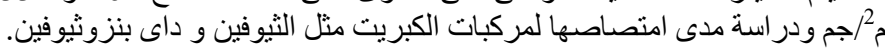

